\title{
Perturbation analysis of a parametrically changed sine-Gordon equation
}

\author{
Sakai, S.; Samuelsen, Mogens Rugholm; OIsen, O. H.
}

Published in:

Physical Review B

Link to article, DOI:

10.1103/PhysRevB.36.217

Publication date:

1987

Document Version

Publisher's PDF, also known as Version of record

Link back to DTU Orbit

Citation (APA):

Sakai, S., Samuelsen, M. R., \& Olsen, O. H. (1987). Perturbation analysis of a parametrically changed sineGordon equation. Physical Review B, 36(1), 217-225. https://doi.org/10.1103/PhysRevB.36.217

\section{General rights}

Copyright and moral rights for the publications made accessible in the public portal are retained by the authors and/or other copyright owners and it is a condition of accessing publications that users recognise and abide by the legal requirements associated with these rights.

- Users may download and print one copy of any publication from the public portal for the purpose of private study or research.

- You may not further distribute the material or use it for any profit-making activity or commercial gain

- You may freely distribute the URL identifying the publication in the public portal

If you believe that this document breaches copyright please contact us providing details, and we will remove access to the work immediately and investigate your claim 


\title{
Perturbation analysis of a parametrically changed sine-Gordon equation
}

\author{
S. Sakai* and M. R. Samuelsen \\ Physics Laboratory I, The Technical University of Denmark, DK-2800, Lyngby, Denmark
}

O. H. Olsen

Protein Engineering Department, NOVO Research Institute, Novo Alle, DK-2880 Bagsvaerd, Denmark

(Received 2 February 1987)

\begin{abstract}
A long Josephson junction with a spatially varying inductance is a physical manifestation of a modified sine-Gordon equation with parametric perturbation. Soliton propagation in such Josephson junctions is discussed. First, for an adiabatic model where the inductance changes smoothly compared with soliton size, transmission or reflection of the soliton is described using a simple energy analysis. Next, the soliton propagation is solved on the basis of a perturbation theory constructed by McLaughlin and Scott. Radiation as well as soliton trajectories are presented numerically. Agreement between such solutions and the results of direct numerical integration by means of a finitedifference method is excellent.
\end{abstract}

\section{INTRODUCTION}

In this paper, a parametrically changed sine-Gordon system is investigated. As is known well, a good physical manifestation of the sine-Gordon system is a long Josephson junction. ${ }^{1}$ The parametrically changed system treated here corresponds to a long Josephson junction whose inductance is a spatially varying function. To examine soliton behavior in such a system is quite significant not only in fundamental studies but also in practical applications, because reflection or trapping of a soliton in the inductance-changed sine-Gordon system is an attractive object for data-processing applications which have been proposed since the 1970 s. $^{2}$ In a recent experiment on a fluxon device, the inductance-changed long Josephson junction was made a potential well for a fluxon losing its velocity by means of an external shunt resistor. ${ }^{3}$

In this paper, the soliton propagation is described on the basis of perturbation analysis. First, a simple energy analysis is performed for the adiabatic model. Next, by applying a general perturbation theory constructed by McLaughlin and Scott ${ }^{4}$ to our system, not only time modulation of soliton parameters (i.e., the velocity and position parameters) but also the radiation (the first-order correction of the perturbation) are evaluated. The results are compared with results of direct numerical integrations of the partial differential equation. The agreement is excellent.

\section{SYSTEM DESCRIPTION}

If the loss terms of the junction are neglected, the fundamental equations for describing a long Josephson junction (Josephson transmission line or JTL) with the inductance $l(x)$ are given by

$$
\begin{aligned}
& \frac{\partial V}{\partial x}=-l(x) \frac{\partial I}{\partial t}, \\
& \frac{\partial I}{\partial x}=-\frac{\partial V}{\partial t}-\sin \phi,
\end{aligned}
$$

$$
\frac{\partial \phi}{\partial t}=V
$$

where the following normalized units are used: $V$ is the voltage across the junction normalized to $\left(\hbar I_{0} / 2 e C\right)^{1 / 2}, I$ is the current flowing along the line normalized to $\left(\hbar I_{0} / 2 e L_{1}\right)^{1 / 2}, \phi$ is the phase difference between two superconducting electrodes, $x$ is the distance measured along the JTL normalized to $\left(\hbar / 2 e L_{1} I_{0}\right)^{1 / 2}, t$ is the time normalized to $\left(\hbar C / 2 e I_{0}\right)^{1 / 2}, C$ is the capacitance of the junction per unit length, $I_{0}$ is the maximum supercurrent per unit length, and $L_{1}$ is the inductance per unit length as $x \rightarrow-\infty$. The function $l(x)$ is assumed to have asymptotic values at $x \rightarrow \pm \infty$, and is normalized to the asymptotic value, $L_{1}$ at $x \rightarrow-\infty$, i.e.,

$$
l(x) \rightarrow\left\{\begin{array}{l}
1 \text { as } x \rightarrow-\infty, \\
l_{0} \text { as } x \rightarrow \infty .
\end{array}\right.
$$

We get, from Eqs. (1a) and (1c),

$$
\phi_{x}=-l(x) I
$$

and from Eqs. (3), (1b), and (1c),

$$
-\partial_{x}\left(\frac{1}{l(x)} \phi_{x}\right)+\phi_{t t}+\sin \phi=0 \text {. }
$$

Hereafter in the present paper, partial derivatives are designated such as $\partial_{x} A$ or $A_{x}$, etc. One approach to the perturbation analysis is that we rearrange Eq. (4) as

$$
-\phi_{x x}+\phi_{t t}+\sin \phi=-\phi_{x x}+\partial_{x}\left(\frac{1}{l(x)} \phi_{x}\right)
$$

and treat the right-hand side of Eq. (4') as the perturbation. Then it is natural that the trial function (or the zeroth-order solution in the perturbation series) is chosen as

$$
\phi=4 \tan ^{-1} \exp \left[\frac{x-X(t)}{\left[1-u^{2}(t)\right]^{1 / 2}}\right),
$$


with

$$
X(t)=\int_{0}^{t} u\left(t^{\prime}\right) d t^{\prime}+x_{0}(t),
$$

where slow time modulations of the parameters $u$ and $x_{0}$ are allowed. Equation (5), which includes the asymptotic form as $x \rightarrow-\infty$, however, never includes the following asymptotic form as $x \rightarrow \infty$ :

$$
\phi=4 \tan ^{-1} \exp \left[\left(\frac{l_{0}}{1-l_{0} u_{f}^{2}}\right)^{1 / 2}\left(x-u_{f} t-x_{1}\right)\right],
$$

where $u_{f}$ is a final velocity and $x_{1}$ is a constant.

To avoid this defect, a variable transformation is performed, i.e.,

$$
d z=l(x)^{1 / 2} d x .
$$

Then, Eq. (4) is expressed as

$$
-\phi_{z z}+\phi_{t t}+\sin \phi=-\frac{1}{2} \partial_{z}[\ln l(x)] \phi_{z} .
$$

Since the perturbing term of Eq. (7) approaches zero as $z \rightarrow \pm \infty$, Eq. (7) indicates that the "two media problem with transient region" is replaced by "impurity problems in one medium." It is apparent that in this case the trial function expressed by $z$ and $t$, corresponding to Eq. (5), fulfills the asymptotic condition as $z \rightarrow \infty$.

\section{ENERGY ANALYSIS}

Before discussing the perturbation analysis, the simple energy analysis is described. Since the energy stored in the inductance is a function of $x$, the total energy $H$ of the system is expressed as

$$
H=\int_{-\infty}^{\infty}\left[\frac{1}{2} \phi_{t}^{2}+\frac{1}{2 l(x)} \phi_{x}^{2}+1-\cos \phi\right) d x
$$

or

$$
H=\int_{-\infty}^{\infty} l(x)^{-1 / 2}\left(\frac{1}{2} \phi_{t}^{2}+\frac{1}{2} \phi_{z}^{2}+1-\cos \phi\right) d z .
$$

Let us consider the system where $l(x)$ varies slowly compared with the spatial size of a soliton. In that system, the energy of a soliton is conserved, because radiation can be neglected and the system is nondissipative. So, for such an adiabatic model, simple energy analysis is possible.

If a soliton has an initial velocity $v_{i}$ and exists at $z<<$, the solution is

$$
\phi=4 \tan ^{-1} \exp \left[\gamma\left(v_{i}\right)\left(z-v_{i} t-z_{1}\right)\right],
$$

where the Lorentz factor $\gamma$ is a function of velocity $v$ as

$$
\gamma(v)=\left(1-v^{2}\right)^{-1 / 2}
$$

and $z_{1}$ is a constant. Putting Eq. (9) into Eq. ( $\left.8^{\prime}\right)$, the initial energy $H_{i}$ at $z<<0$ is ${ }^{4}$

$$
H_{i}=8 \gamma\left(v_{i}\right) \text {. }
$$

If the soliton is transmitted to the region at $z \gg 0$, the final energy $H_{f}$ with the final velocity $v_{f}$ on the $z$ axis is obtained similarly as

$$
H_{f}=8 l_{0}^{-1 / 2} \gamma\left(v_{f}\right) .
$$

Thus the condition that the soliton is transmitted is

$$
\gamma\left(v_{i}\right)>l_{0}^{-1 / 2} \gamma(0)=l_{0}^{-1 / 2}
$$

or

$$
v_{i}^{2}>1-l_{0},
$$

which leads to the following summary.

(i) When $l_{0}>1$, a soliton with $v_{i}\left(0<v_{i}<1\right)$ is always transmitted to the region, $z>0$.

(ii) When $0<l_{0}<1$, two cases occur, i.e., if $v_{i}>\left(1-l_{0}\right)^{1 / 2}$, the soliton is transmitted, but if $0<v_{i}<\left(1-l_{0}\right)^{1 / 2}$, it is reflected. From $H_{i}=H_{f}$, the velocity relations between the initial and final states are for the transmission case,

$$
v_{f}=\left[1-l_{0}^{-1}\left(1-v_{i}^{2}\right)\right]^{1 / 2}
$$

and for the reflection cases,

$$
v_{f}=-v_{i} \text {. }
$$

It is obvious from Eq. (6) that the velocity $u$ on the $x$ axis is connected to $v$ on the $z$ axis for the limits at $x= \pm \infty$ as

$$
u=\left\{\begin{array}{l}
v \text { at } x=-\infty, \\
l_{0}^{-1 / 2} v \text { at } x=\infty .
\end{array}\right.
$$

\section{PERTURBATION ANALYSIS}

When $l(x)$ is not a slowly varying function of $x$, a more general treatment is needed, because radiation (e.g., the distortion of soliton shapes, tailing or reflection) exists. The general perturbation theory reported by McLaughlin and Scott $^{4}$ is applied to our system.

\section{A. McLaughlin and Scott theory}

In this section, the perturbation theory in Ref. 4 is reviewed. The purpose of this review is to elucidate the relationship between the theory and the actual numerical treatment described in Sec. IV B. The formalism is written for a single soliton case in spite of the fact that the theory ${ }^{4}$ is more general.

\section{Preparation}

Another representation of Eq. (7) is

$$
\left(\begin{array}{cc}
\partial_{t} & -1 \\
-\partial_{z z}+\sin (\cdots) & \partial_{t}
\end{array}\right)\left(\begin{array}{c}
\phi \\
\phi_{t}
\end{array}\right)=\left(\begin{array}{c}
0 \\
\epsilon f
\end{array}\right),
$$

where $\epsilon f$ is the perturbation and in this case

$$
\epsilon f=-\frac{1}{2} \partial_{z}[\ln l(x)] \phi_{z}
$$

and the solution $\operatorname{col}\left(\phi, \phi_{t}\right)$ is of the form

$$
\left(\begin{array}{l}
\phi \\
\phi_{t}
\end{array}\right)=\left(\begin{array}{l}
\phi^{(0)} \\
\phi_{t}^{(0)}
\end{array}\right)+\epsilon\left[\begin{array}{l}
\phi^{(1)} \\
\phi_{t}^{(1)}
\end{array}\right)+\cdots .
$$

Here the first term of Eq. (17), which is the zeroth-order 
perturbing term, expresses the soliton profile where soliton parameters are allowed to modulate slowly as a function of $t$, i.e.,

$$
\left[\begin{array}{c}
\phi^{(0)} \\
\phi_{t}^{(0)}
\end{array}\right]=\left(\begin{array}{c}
4 \tan ^{-1} \exp \theta \\
-2 \gamma[v(t)] v(t) \operatorname{sech} \theta
\end{array}\right)
$$

with

$$
\begin{aligned}
& \theta=\gamma[v(t)][z-Z(t)], \\
& Z(t)=\int_{0}^{t} v\left(t^{\prime}\right) d t^{\prime}+z_{0}(t) .
\end{aligned}
$$

The second term of Eq. (17), being the first-order correction, must fulfill the following equations using the zerothorder solution:

$$
\left(\begin{array}{cc}
\partial_{t} & -1 \\
-\partial_{z z}+\cos \phi^{(0)} & \partial_{t}
\end{array}\right)\left(\begin{array}{l}
\phi^{(1)} \\
\phi_{t}^{(1)}
\end{array}\right)=\left(\begin{array}{l}
F_{1} \\
F_{2}
\end{array}\right)
$$

where

$$
\left(\begin{array}{l}
F_{1} \\
F_{2}
\end{array}\right]=\left(\begin{array}{c}
0 \\
f\left[\phi^{(0)}\right]
\end{array}\right]-\frac{1}{\epsilon}\left[\begin{array}{l}
\phi_{v}^{(0)} \frac{d v}{d t}+\phi_{z_{0}}^{0} \frac{d z_{0}}{d t} \\
\phi_{t v}^{(0)} \frac{d v}{d t}+\phi_{t z_{0}}^{(0)} \frac{d z_{0}}{d t}
\end{array}\right]
$$

with

$$
\begin{aligned}
& \phi_{v}^{(0)}=2 \gamma^{2} v \theta \operatorname{sech} \theta, \\
& \phi_{z_{0}}^{(0)}=-2 \gamma \operatorname{sech} \theta, \\
& \phi_{t v}^{(0)}=2 \gamma^{3} \operatorname{sech} \theta\left(v^{2} \theta \tanh \theta-1\right), \\
& \phi_{t z_{0}}^{(0)}=-2 \gamma^{2} v \operatorname{sech} \theta \tanh \theta .
\end{aligned}
$$

Equation (21) consists not only of $f\left[\phi^{(0)}\right]$ but also of the effective source induced by the time modulation of the soliton parameters. In Eq. $(21), \phi_{v}^{(0)}$ and $\phi_{t v}^{(0)}$ means that, when differentiating $\phi^{(0)}$ and $\phi_{t}^{(0)}$ with respect to $v, Z(t)$ is fixed.

\section{Time evolution of the soliton parameters}

The modulation of the soliton parameters can be evaluated without the knowledge of the soliton of the firstorder term, Eq. (20). According to the theory in Ref. 4, the parameters are modulated to avoid linear growth of $\phi^{(1)}$ with the increase of $t$. This is realized when $f$ is orthogonal to the discrete null space of the adjoint operator $L^{+}$, where

$$
L^{+}=-\left(\begin{array}{cc}
\partial_{t} & \partial_{z z}-\cos \phi^{(0)} \\
1 & \partial_{t}
\end{array}\right)
$$

and for a single soliton case, the discrete null space has two bases, i.e., $\operatorname{col}\left[\phi_{t v}^{(0)},-\phi_{v}^{(0)}\right]$ and $\operatorname{col}\left[\phi_{t z_{0}}^{(0)},-\phi_{z_{0}}^{(0)}\right]$. Consequently, soliton parameters obey the following coupled ordinary differential equations (ODE):

$$
\begin{aligned}
& \frac{d v}{d t}=-\epsilon \frac{1}{4 \gamma^{2}} \int_{-\infty}^{\infty} f\left[\phi^{(0)}\right] \operatorname{sech} \theta d z, \\
& \frac{d z_{0}}{d t}=-\epsilon \frac{v}{4 \gamma} \int_{-\infty}^{\infty} f\left[\phi^{(0)}\right] \theta \operatorname{sech} \theta d z .
\end{aligned}
$$

\section{The first-order correction}

After the time evolution of the soliton parameters is known, the first-order correction can be evaluated by

$$
\left[\begin{array}{l}
\phi^{(1)} \\
\phi_{t}^{(1)}
\end{array}\right]=\int_{0}^{t} d t^{\prime} \int_{-\infty}^{\infty} d z^{\prime} G_{c}\left(z, t \mid z^{\prime}, t^{\prime}\right) F\left(z^{\prime}, t^{\prime}\right)
$$

where

$$
F=\operatorname{col}\left(F_{1}, F_{2}\right)
$$

and

$$
G_{c}\left(z, t \mid z^{\prime}, t^{\prime}\right)=-\frac{\pi i}{4} \int_{-\infty}^{\infty} \frac{\lambda d \lambda}{a^{2}(\lambda)}\left[\begin{array}{ll}
\Psi^{+}(z, t) \Psi_{t^{\prime}}^{-}\left(z^{\prime}, t^{\prime}\right) & -\Psi^{+}(z, t) \Psi^{-}\left(z^{\prime}, t^{\prime}\right) \\
\Psi_{t}^{+}(z, t) \Psi_{t^{\prime}}^{-}\left(z^{\prime}, t^{\prime}\right) & -\Psi_{t}^{+}(z, t) \Psi^{-}\left(z^{\prime}, t^{\prime}\right)
\end{array}\right)
$$

$\Psi^{ \pm}$are the solution to

$$
\left[\partial_{z z}-\partial_{t t}-\cos \phi^{(0)}\right] \Psi^{ \pm}=0
$$

and satisfy the boundary conditions

$$
\Psi^{ \pm} \rightarrow \frac{1}{\pi \lambda} \exp \{\mp i[k(\lambda) z+\omega(\lambda) t]\} \text { as } z \rightarrow \mp \infty,
$$

with

$$
\begin{aligned}
& k(\lambda)=2 \lambda-\frac{1}{8 \lambda}, \\
& \omega(\lambda)=2 \lambda+\frac{1}{8 \lambda}, \\
& \omega^{2}=k^{2}+1 .
\end{aligned}
$$

After the correction of typographical errors appearing in Ref. 4, the solutions for a single soliton case are expressed explicitly: $\Psi^{ \pm}$are given by

$\Psi^{+}=\frac{1}{\pi \lambda} \frac{\lambda^{2}+\zeta^{2}-2 \lambda \zeta \tanh \theta}{(\lambda+\zeta)^{2}} \exp \{-i[k(\lambda) z+\omega(\lambda) t]\}$

and

$\Psi^{-}=\frac{1}{\pi \lambda} \frac{\lambda^{2}+\zeta^{2}+2 \lambda \zeta \tanh \theta}{(\lambda+\zeta)^{2}} \exp \{i[k(\lambda) z+\omega(\lambda) t]\}$,

with 


$$
\begin{aligned}
& a(\lambda)=(\lambda-\zeta)(\lambda+\zeta)^{-1}, \\
& \zeta=\frac{i}{4}\left(\frac{1-u}{1+u}\right)^{1 / 2},
\end{aligned}
$$

$$
G_{c}=\frac{1}{4 \pi i} \int_{-\infty}^{\infty} d \lambda \frac{\exp \left\{-i\left[k(\lambda)\left(z-z^{\prime}\right)+\omega(\lambda)\left(t-t^{\prime}\right)\right]\right\}}{\lambda\left(\xi^{2}-\lambda^{2}\right)^{2}}\left(\begin{array}{ll}
A B & -A C \\
D B & -D C
\end{array}\right),
$$

where

$$
\begin{aligned}
& A=\zeta^{2}+\lambda^{2}-2 \lambda \zeta \tanh \theta(z, t), \\
& B=i \omega C-2 i \lambda \zeta \omega(\zeta) \operatorname{sech}^{2} \theta\left(z^{\prime}, t^{\prime}\right), \\
& C=\zeta^{2}+\lambda^{2}+2 \lambda \zeta \tanh \theta\left(z^{\prime}, t^{\prime}\right), \\
& D=-i \omega A+2 i \lambda \zeta \omega(\zeta) \operatorname{sech}^{2} \theta(z, t) .
\end{aligned}
$$

\section{B. Application of the McLaughlin and Scott theory}

The theory described in the last section is applied to our system.

\section{Time evolution of the soliton parameters}

Putting Eq. (16) into Eq. (24) and using Eq. (19b), we get the ODE for the soliton parameters:

$$
\begin{aligned}
& \frac{d v}{d t}=\frac{1}{4 \gamma} \int_{-\infty}^{\infty} \partial_{z}[\ln l(x)] \operatorname{sech}^{2} \theta d z, \\
& \frac{d Z}{d t}=v+\frac{v}{4} \int_{-\infty}^{\infty} \partial_{z}[\ln l(x)] \theta \operatorname{sech}^{2} \theta d z .
\end{aligned}
$$

Next, we give two concrete functional forms for $l(x)$ and investigate the time modulation of the soliton parameters for each case.

(i) When $l(x)$ changes abruptly from 1 to $l_{0}$ at $x=0$, as shown in Fig. 1(a), we have

$$
\partial_{z}[\ln l(x)]=\left(\ln l_{0}\right) \delta(z),
$$

resulting in

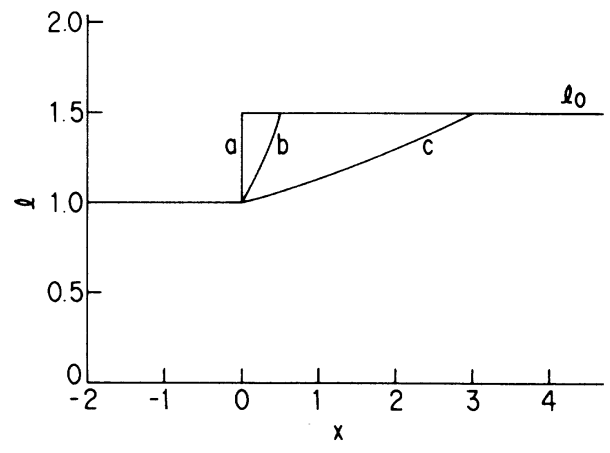

FIG. 1. Spatially varying inductance, $l(x)$. Curve $a$ is a function with a step change at $x=0$. Curves $b$ and $c$ are gradually changed $l(x)$ obeying Eq. (42).

$$
\begin{aligned}
& \frac{d v}{d t}=\frac{1}{4 \gamma}\left(\ln l_{0}\right) \operatorname{sech}^{2}(\gamma Z), \\
& \frac{d Z}{d t}=v-\frac{1}{4}\left(\ln l_{0}\right) v \gamma Z \operatorname{sech}^{2}(\gamma Z) .
\end{aligned}
$$

In Figs. 2 the velocity parameter $v(t)$ versus the position parameter, $Z(t)=\int_{-\infty}^{\infty} v d t^{\prime}+z_{0}(t)$, is shown for various initial velocities. Figure $2(a)$ is the result when $l_{0}=1.5$ and Fig. 2(b) is the result when $l_{0}=0.6$. As shown in the figures, the soliton always propagates to the right side for $l_{0}=1.5$, but for $l_{0}=0.6$, if the initial velocities are small, the soliton is reflected. These results are
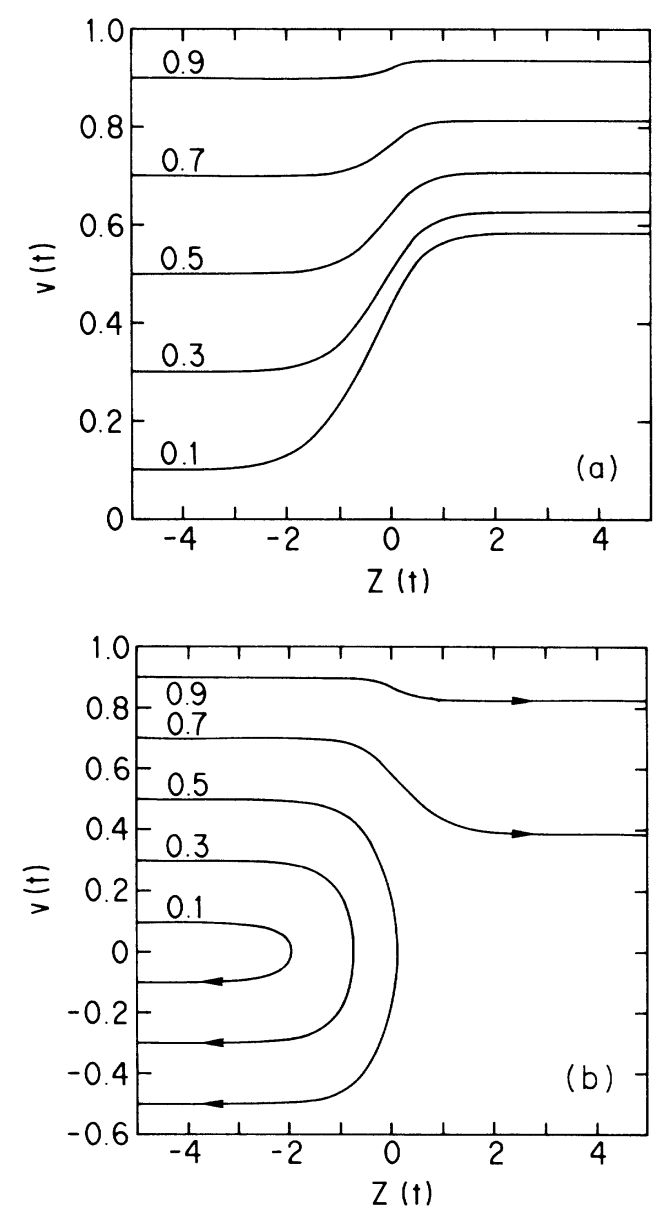

FIG. 2. $v(t)$ vs $Z(t)$ when $l(x)$ is a step function. (a) $l_{0}=1.5$ and (b) $l_{0}=0.6$. The number denoted in each curve is the initial velocity. 
the same as those of the adiabatic energy analysis in Sec. III. Figure 3 shows the relation on the $x$ axis between the velocity parameter $u(t)$ and the position parameter $X(t)$ for $l_{0}=1.5$, where $X(t)$ is connected to the position $Z(t)$ on the $z$ axis by

$$
X(t)=\left\{\begin{array}{l}
Z(t) \text { for } Z \leq 0 \text { or } X \leq 0, \\
l_{0}^{-1 / 2} Z(t) \text { for } Z>0 \text { or } X>0 .
\end{array}\right.
$$

Since the ratio of $-\phi_{t}^{(0)}$ to $\phi_{x}^{(0)}$ is generally a function of $x$, $u(t)$ must be defined carefully. Here we define $u$ as that ratio at the soliton position, i.e.,

$$
u \equiv-\left.\frac{\phi_{t}^{0}}{\phi_{x}^{(0)}}\right|_{x=X(t)}=l(x)^{-1 / 2} v=\left\{\begin{array}{l}
v \text { for } X \leq 0 \\
l_{0}^{-1 / 2} v \text { for } X>0
\end{array}\right.
$$

As shown in Fig. 3, for $l_{0}>1$, we find that (1) there exist abrupt changes of $u$ resulting from the jump of $l(x)$; (2) a soliton with high initial velocity has a decreased final velocity but, on the other hand, a soliton with low initial velocity has an increased one. The energetic point of view similar to that in the adiabatic model [Eq. (13a)] is still helpful in order to understand this fact. In Fig. 4 the time dependence of the soliton position resulting from the perturbation analysis is shown (solid curve). Solid circles (๑) in Fig. 4 are the result of direct numerical integration of the partial differential equation, Eq. (4). Good agreement is found. Here, the numerical integration is performed by a standard finite-difference method based on a stabilized leapfrog scheme. The abrupt change of $l(x)$ is treated as $1 / l(0) \simeq\left(1+l / l_{0}\right) / 2$ and $\left.\partial_{x}[1 / l(x)]\right|_{x=0}$ $\left.\simeq\left(1 / l_{0}\right)-1\right) / \Delta x$, where $\Delta x$ is the spatial stepsize $(=0.0025)$. The time step is equal to the spatial stepsize. The soliton position is taken from the $x$ value at which $\phi_{x} / l(x)$ has the maximum.

(ii) Next, let us consider the case where $l(x)$ changes gradually as shown in curves in Figs. 1(b) and (c). It is practically convenient to choose $l(x)$ so that Eqs. (37) is

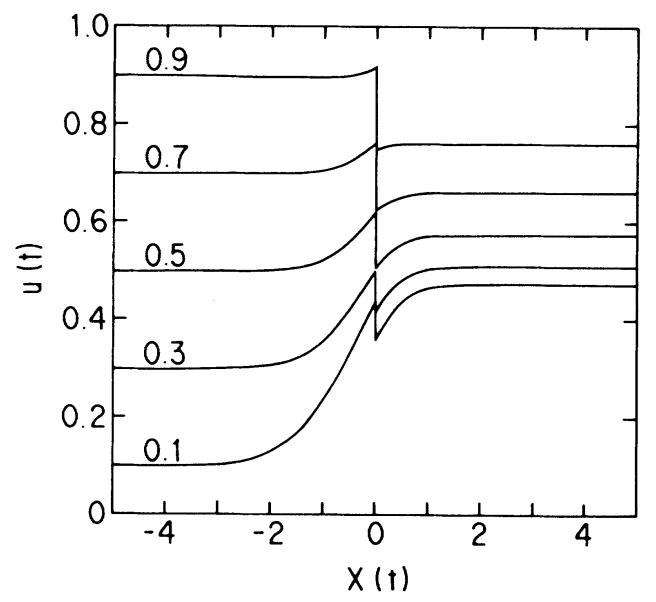

FIG. 3. $u(t)$ vs $X(t)$ when $l(x)$ is a step function and $l_{0}=1.5$. The number denoted in each curve is the initial velocity. integrable by $z$. One example of the integrable form is

$$
l(x)=\left\{\begin{array}{l}
1 \text { for } x \leq 0, \\
{\left[1-\frac{h}{2} x\right]^{1 / 2} \quad \text { for } 0<x \leq a} \\
l_{0} \text { for } a<x,
\end{array}\right.
$$

with

$$
h=\frac{2}{a}\left(1-l_{0}^{-1 / 2}\right) .
$$

Using Eq. (42) and integrating $d z / d x=l(x)^{1 / 2}$, we get the relation between $z$ and $x$

$$
z=\left\{\begin{array}{l}
x \text { for } x \leq 0 \text { or } z \leq 0, \\
-\frac{2}{h} \ln \left[1-\frac{h}{2} x\right) \text { for } 0<x \leq a \text { or } 0<z \leq b, \\
l_{0}^{1 / 2} x+b-a l_{0}^{1 / 2} \text { for } a<x \text { or } b<z,
\end{array}\right.
$$

where

$$
b h=\ln l_{0} .
$$

Thus,

$$
\frac{1}{2} \partial_{z}[\ln l(x)]= \begin{cases}0 & \text { for } z \leq 0 \\ \frac{h}{2} & \text { for } 0<z \leq b \\ 0 & \text { for } b<z\end{cases}
$$

is obtained, and the right-hand side of Eq. (37) is integrated as

$$
\begin{aligned}
& \frac{d v}{d t}=-\frac{h}{4 \gamma^{2}}\{\tanh [\gamma(Z-b)]-\tanh (\gamma Z)\} \\
& \frac{d Z}{d t}=v+\frac{h v}{4 \gamma}[\gamma(Z-b) \tanh [\gamma(Z-b)]-\gamma Z \tanh (\gamma Z) \\
& \left.+\ln \left(\frac{\cosh (\gamma Z)}{\cosh [\gamma(Z-b)]}\right)\right]
\end{aligned}
$$

From this example, we can see how the soliton propaga-

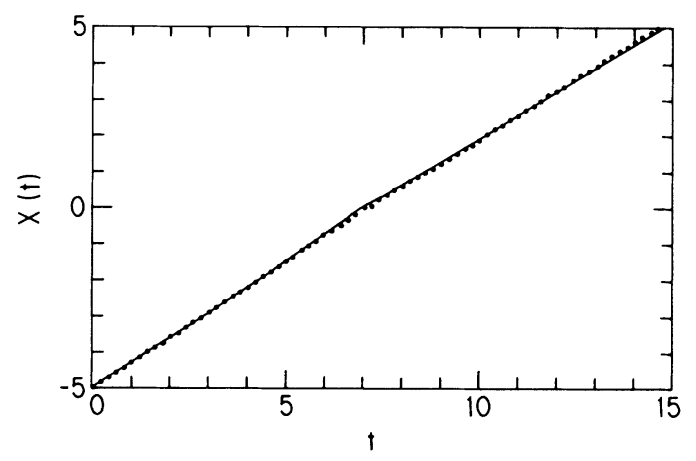

FIG. 4. The soliton position $X$ as a function of time for the abruptly changed $l(x)$, where $u_{i}=v_{i}=0.7$ and $l_{0}=1.5$. The solid curve is the result of the perturbation analysis and the solid circles (๑) are those of the direct numerical integration of Eq. (4) by means of the finite-difference method. 
tion depends on the smoothness of $l(x)$. Note that Eq. (46) includes both the abrupt limit [Fig. 1(a)] and the smooth limit (adiabatic model). The former is easily found [Eq. (39)] by taking $b \rightarrow 0$ and holding $b h=\ln l_{0}$ (constant). Figure 5 shows the velocity versus the position on the $x$ axis for various values of $a$. The result is reasonable, i.e., the overshoot of the velocity around the transient region is small with smoothing $l(x)$.

\section{The zeroth-order energy}

Let us consider the zeroth-order energy, $H^{(0)}(t)$ calculated from the zeroth-order solution. Inserting Eq. (18) into Eq. $\left(8^{\prime}\right)$ we get

$$
H^{(0)}(t)=4 \gamma^{2} \int_{-\infty}^{\infty} l(x)^{-1 / 2} \operatorname{sech}^{2} \theta d z
$$

As already discussed in Sec. III, $H^{(0)}$ is proportional to the Lorentz factor $\gamma$ as $t \rightarrow \pm \infty$, i.e.,

$$
\begin{aligned}
& H^{(0)}(-\infty)=8 \gamma[u(-\infty)], \\
& H^{(0)}(\infty)=8 l_{0}^{-1 / 2} \gamma[u(\infty)] .
\end{aligned}
$$

As far as discussion is limited at $t \rightarrow \pm \infty$, it is enough to investigate $\gamma$ instead of $H^{(0)}$. From Eq. (37), we get

$$
\frac{d \gamma}{d t}=\gamma^{3} v \frac{d v}{d t}=\frac{\gamma}{4} \int_{-\infty}^{\infty} \partial_{z}[\ln l(x)] \gamma v \operatorname{sech}^{2} \theta d z
$$

Using

$$
\partial_{t}(\tanh \theta)=\operatorname{sech}^{2} \theta\left(\gamma^{2} v \theta \frac{d v}{d t}-\gamma \frac{d z_{0}}{d t}\right)-\gamma v \operatorname{sech}^{2} \theta
$$

and Eq. (37) again, we get

$$
\frac{d}{d t}(\ln \gamma)=-\frac{1}{4} \partial_{t} \int_{-\infty}^{\infty} \partial_{z}[\ln l(x)] \tanh \theta d z
$$

In the condition that a soliton is transmitted, we have $\tanh \theta \rightarrow 1$ and $l(x) \rightarrow 1$ when $t \rightarrow-\infty$, and $\tanh \theta \rightarrow-1$, and $l(x) \rightarrow l_{0}$ when $t \rightarrow \infty$. So, the integration of Eq. (49) leads to

$$
\gamma[u(-\infty)]=l_{0}^{-1 / 2} \gamma[u(\infty)] .
$$

This means from Eq. (48) that

$$
H^{(0)}(-\infty)=H^{(0)}(\infty) .
$$

When the soliton is reflected, $\tanh \theta \rightarrow 1$ and $l(x) \rightarrow 1$ as $t \rightarrow \infty$. We get $\quad \gamma[u(-\infty)]=\gamma[u(\infty)]$, i.e., $H^{(0)}(-\infty)=H^{(0)}(\infty)$. So, the asymptotic values of the zeroth-order energy as $t \rightarrow \infty$ are preserved, regardless of function shapes of $l(x)$ and the conditions of transmission or reflection. However, this surprising result does not

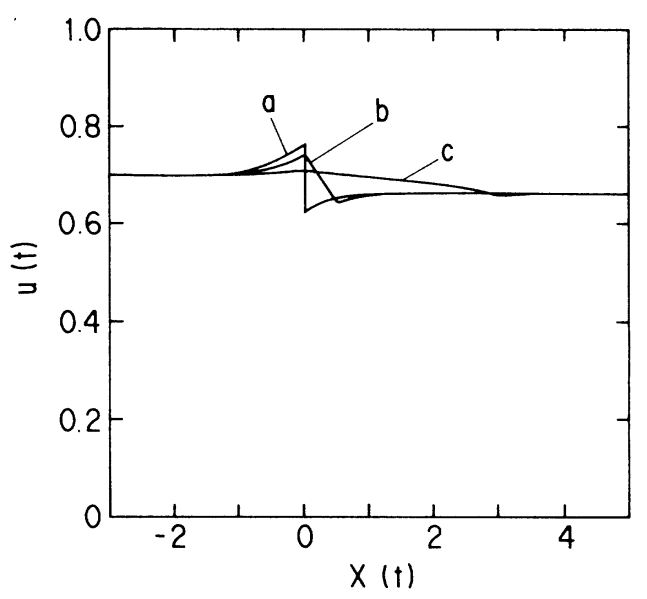

FIG. 5. Soliton trajectories [ $u(t)$ vs $X(t)$ ] in JTL's with gradually changed $l(x)$ as Eq. (42) where $l_{0}=1.5$ and $u_{i}=v_{i}=0.7$. The curves $a, b$, and $c$ are the results for the three different lengths of the transient region, i.e., $a=0,0.5$, and 3 , respectively.

mean that there are no radiations at $t \rightarrow \infty$. In fact, the direct numerical integrations of Eq. (4) show that there exist radiations. Figure 6 shows the reflected radiation energy versus $l_{0}$ for various initial velocities, $u_{i}$ when the abrupt change of $l(x)$ at $x=0$ is assumed. Here the reflected radiation energy is defined by the integration of the energy density from $-\infty$ to 0 well after the soliton passes through the boundary, $x=0$. Figure 6 suggests that, in spite of getting the relation, $H^{(0)}(-\infty)=H^{(0)}(\infty)$, we need discussions including the higher-order corrections as will be treated in IV B 4.

However, the fact that, whatever function $l(x)$ is, the zeroth-energy order is preserved at two limits, $t \rightarrow \pm \infty$, is convenient practically, because without calculating not only the first-order correction but also the zeroth-order ODE [Eq. (37)] we may know the final velocity of the zeroth-order soliton trajectories using the simple adiabatic model described in Sec. III.

\section{The first-order correction}

The numerical example described here is for the model that $l(x)$ changes abruptly at $x=0$ as shown in Fig. 1(a). The calculation procedure for the theory reviewed in IV A 2 is as follows. By changing the order of integrations in Eqs. (25) and (26) and by changing the variable from $\lambda$ to $k$, we obtain

$$
\left(\begin{array}{l}
\phi^{(1)} \\
\phi_{t}^{(1)}
\end{array}\right)=\frac{1}{2 \pi} \int_{-\infty}^{\infty} d k \int_{0}^{t} d t^{\prime} \int_{-\infty}^{\infty} d z^{\prime} \operatorname{Im}\left[\frac{\exp \left\{-i\left[k\left(z-z^{\prime}\right)+\omega\left(t-t^{\prime}\right)\right]\right\}}{\omega\left(\zeta^{2}-\lambda^{2}\right)^{2}}\left[\begin{array}{ll}
A B & -A C \\
D B & -D C
\end{array}\right]\left[\begin{array}{l}
F_{1} \\
F_{2}
\end{array}\right]\right),
$$

with

$$
\begin{aligned}
& \lambda=\frac{1}{4}\left[k+\left(k^{2}+1\right)^{1 / 2}\right] \\
& \omega=\left(k^{2}+1\right)^{1 / 2} .
\end{aligned}
$$

As found in Eqs. (21) and (38) there is a $\delta$ function on the $z$ axis, corresponding to the jump of $l(x)$ at $x=0$. Thus the integration of this $\delta$-function part in Eq. (50) with respect to $z^{\prime}$ and $t^{\prime}$ is replaced by the integration with 


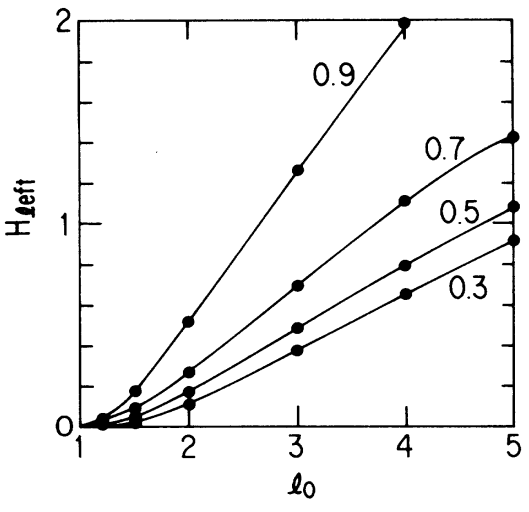

FIG. 6. Radiation energy obtained from the direct numerical integration of Eq. (4). The figure shows $l_{0}$ dependence of the reflective component of the radiation energy, whose definition is found in the text. The parameter denoted in each curve is the initial velocity.

respect to $t^{\prime}$ only. The remaining parts are integrated using a computation routine DBCQDU (Ref. 5) for twofold integration, where $z^{\prime}$ and $t^{\prime}$ are divided in small segments. The integration ranges of this are determined by neglecting the ranges where $\left|F\left(z^{\prime}, t^{\prime}\right)\right|$ (from which the $\delta$ function part is eliminated) is smaller than a threshold value. Finally, Eq. (50) is integrated by the wave number, $k$, using a mathematical routine DCSQDU. ${ }^{5}$ It can be said in general that, if $k$ is much larger than the spatial size of a soliton, the contribution to $\operatorname{col}\left(\phi^{(1)}, \phi_{t}^{(1)}\right)$ is negligible. Thus appropriate limits, $\pm k_{c}$, exist for the integration by $k$. The validity of the integrations of Eq. (50) is assured by the fact that the results are unchanged even after changing the integration ranges for $z^{\prime}, t^{\prime}$, and $k$, and also the sizes of the segments $\Delta z^{\prime}, \Delta t^{\prime}$, and $\Delta k$.

Figure 7 shows the results of the first-order correction, $\phi^{(1)}$ and $\phi_{t}^{(1)}$ at $l_{0}=1.5$ when $Z(0)=-6$ and $v(0)=0.7$. The segment sizes, $\Delta z^{\prime}, \Delta t^{\prime}$, and $\Delta k$ are $0.25,0.25$, and 0.20 , respectively. The upper and lower limits of the integration by $k$ are 6 and -6 , respectively. As shown in the figure, while a soliton passes around $z=0$, radiations begin to occur. These are not damped, and, roughly speaking, are classified into the distortion part of the soliton, the reflection part and oscillatory part staying around $z=0$.

In Fig. 8, the results of the perturbation analysis are compared with that of the direct numerical integration of Eq. (4) under the same condition as in Fig. 7. The solid circles in Figs. 8(a) and (b) show $\phi^{(0)}+\phi^{(1)}$ and $-\left(\phi_{t}^{(0)}+\phi_{t}^{(1)}\right)$, respectively, of the perturbation analysis. The solid curves in Fig. 8(a) and (b) show $\phi$ and $-\phi_{t}$, respectively, of the direct integrations of Eq. (4). The agreement between the solid circles and the solid curves is surprisingly good.

\section{Energy of radiations}

Putting Eq. (18) into Eq. $\left(8^{\prime}\right), H$ is also expressed by a series as

$$
H=H^{(0)}+\epsilon H^{(1)}+\epsilon^{2} H^{(2)}+\cdots,
$$

where

$$
H^{(0)}=\int_{-\infty}^{\infty} l(x)^{-1 / 2}\left\{\frac{1}{2}\left[\phi_{t}^{(0)}\right]^{2}+\frac{1}{2}\left[\phi_{z}^{(0)}\right]^{2}+1-\cos \phi^{(0)}\right\} d z,
$$
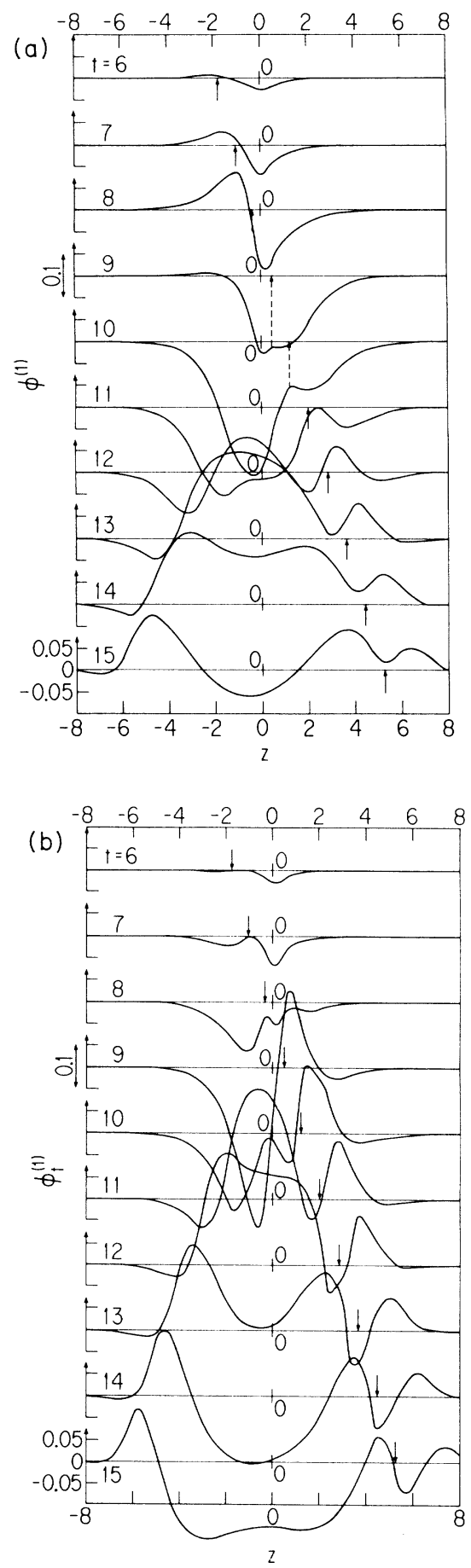

FIG. 7. The first-order radiative correction (a) $\phi^{(1)}$ and (b) $\phi_{t}^{(1)}$ as a function of $z$ at various times. The abrupt changed $l(x)\left(l_{0}=1.5\right)$ is assumed and $v_{i}=0.7, Z(0)=-6.0$. The arrow found in each curve indicates the position of the soliton [i.e., the value of $Z(t)$ ]. 
$H^{(1)}=\int_{-\infty}^{\infty} l(x)^{-1 / 2}\left[\phi_{t}^{(0)} \phi_{t}^{(1)}+\phi_{z}^{(0)} \phi_{z}^{(1)}+\phi^{(1)} \sin \phi^{(0)}\right] d z$

and

$$
\begin{aligned}
H^{(2)}=\int_{-\infty}^{\infty} l(x)^{-1 / 2}[ & \frac{1}{2}\left(\phi_{t}^{(1)}\right)^{2}+\frac{1}{2}\left(\phi_{z}^{(1)}\right)^{2}+\frac{1}{2} \phi^{(1)} \cos \phi^{(0)} \\
& \left.+\phi_{t}^{(0)} \phi_{t}^{(2)}+\phi_{z}^{(0)} \phi_{z}^{(2)}+\phi^{(2)} \sin \phi^{(0)}\right] d z
\end{aligned}
$$

Equations (53) indicate that, if $\operatorname{col}\left(\phi, \phi_{t}\right)$ is solved up to the order of $\epsilon$, there remains uncertainty of $0\left(\epsilon^{2}\right)$ in $H$. In general, if it is solved up to the order of $\epsilon^{i}$, there is uncertainty of $0\left(\epsilon^{i+1}\right)$. Since the system is lossless, $H$ at any time should approach, more and more, the initial energy with taking into account of up to higher-order terms of the series-expanded $\operatorname{col}\left(\phi, \phi_{t}\right)$. Remember the equality re-
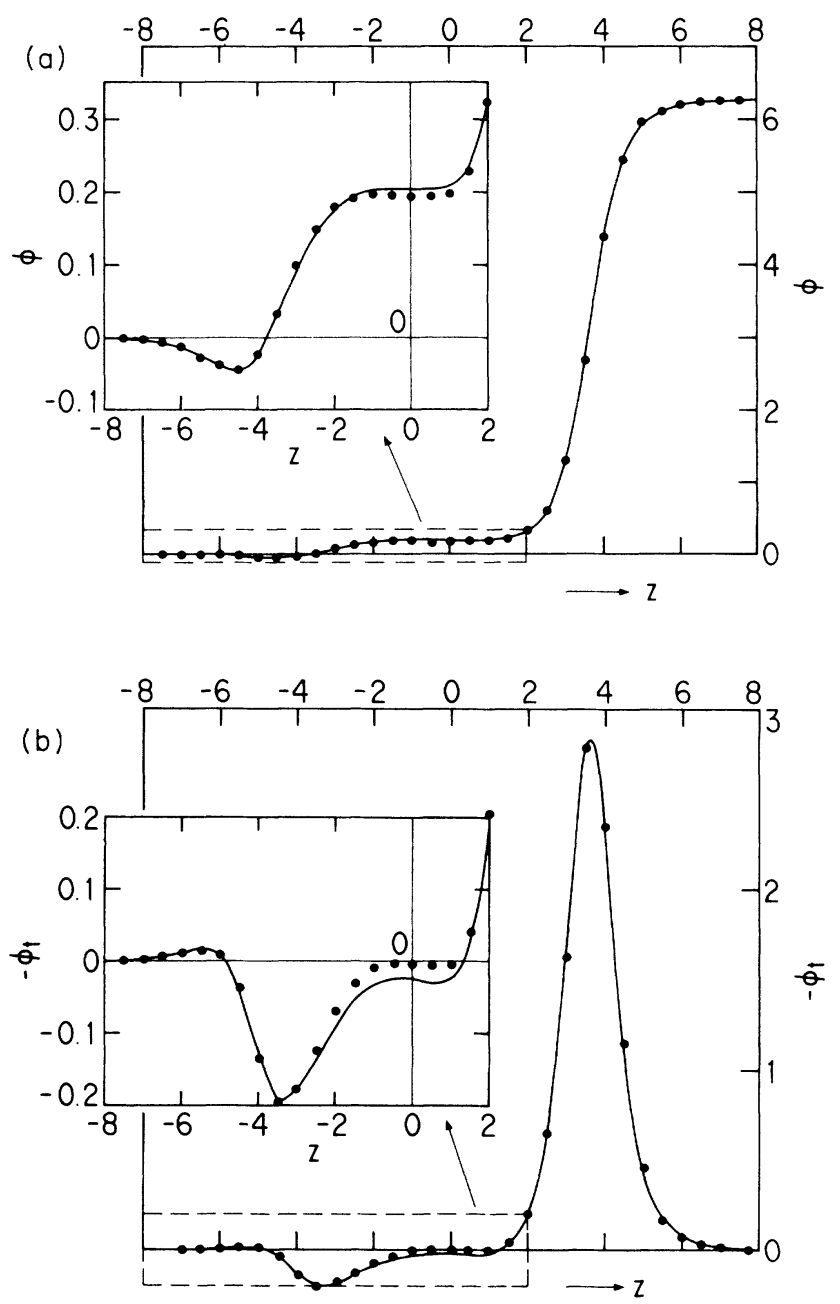

FIG. 8. (a) $\phi(z)$ and (b) $-\phi_{t}(z)$ at $t=13.0$. Solid curves are numerical integration results of Eq. (4) Solid circles (•) represent the perturbation analysis results including the firstorder radiative corrections. The initial conditions at $t=0$ of both methods are $v_{i}=0.7$ and $Z(0)=-6.0$. lation of the zeroth order as $t \rightarrow \pm \infty, H^{(0)}(-\infty)$ $=H^{(0)}(\infty)$, obtained in IV B 2. This comes from the fact that orthogonality conditions to avoid the linear growth of $\phi^{(1)}$ in the McLanghlin and Scott theory are applied to our lossless system. So, this relation does not conflict with the order consideration result of energy discussed here.

A good estimation of radiation is to observe the radiation energy of the "reflection component," which is defined by integrating the energy density in the range from $z=-\infty$ to $z=0$ at some time well after a soliton passes at $z=0$, meaning that the soliton tail is negligible for the range of $z<0$. At this time, since $\phi^{(0)} \simeq 0$ and $\phi_{t}^{(0)} \simeq 0$ between $z=-\infty$ and $z=0$, we get $H_{\text {left }}^{(0)} \simeq 0$ and $H_{\text {left }}^{(1)} \simeq 0$ from Eqs. (53a) and (53b), where $H_{\text {left }}^{(i)}$ is the left half (or the reflection) energy of the $i$ th order. Therefore, $H_{\text {left }}^{(2)}$ is the lowest order of the nonzero reflection energy. Since, as is clear in Eq. $(53 \mathrm{c}), \phi^{(2)}, \phi_{t}^{(2)}$, and $\phi_{z}^{(2)}$ appear in the product forms of the zeroth-order solutions, these are neglected. Thus the reflection energy can be estimated from the first-order solutions even though the energy is second order.

Figure 9 shows the $l_{0}$ dependence of the reflection energy, where the initial velocity is 0.7 , the soliton position at $t=0$ is -6.0 , and the energy observation time is around $t=14-15$ at which point the soliton is far away from $z=0$. In the figure, open circles are the results of the perturbation analysis and plus signs $(+)$ are those of the direct numerical integration of Eq. (4). Agreement for $1<l_{0} \leq 1.5$ between two cases is perfectly good. The relative deviation at $l_{0}=2$ is still as small as 0.01 .

\section{CONCLUSION}

A modified sine-Gordon equation with a parametric perturbation, which is a mathematical model of a lossless

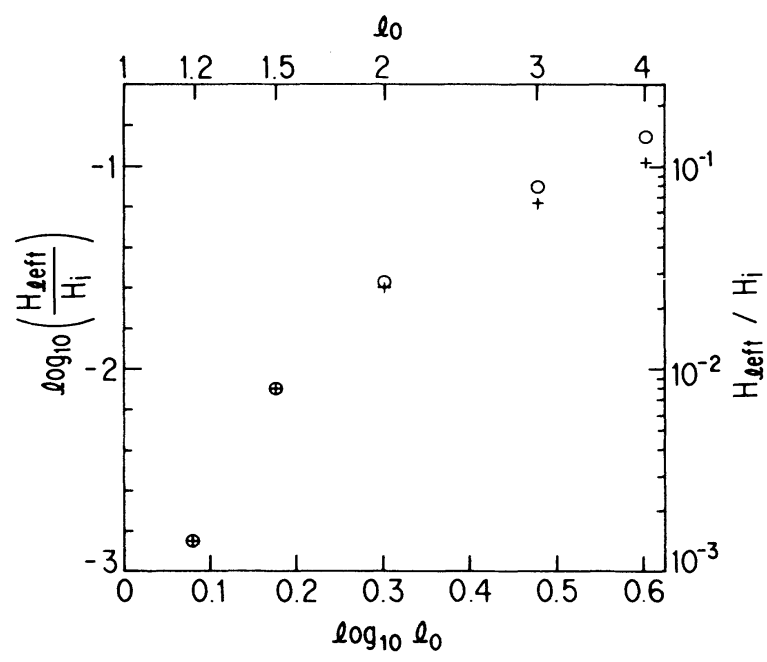

FIG. 9. $l_{0}$ dependence of the reflective component, $H_{\text {left }}$ of radiation energy normalized to the initial soliton energy, $H_{i}$. Symbols $(O)$ and $(+)$ are the perturbation analysis results and the numerical integration results of Eq. (4), respectively. The initial conditions are $v_{i}=0.7$ and $Z(0)=-6.0 . H_{i}$ is calculated from Eq. (11a). 
Josephson transmission line with a spatially varying inductance $l(x)$, has been investigated. The functions $l(x)$ with the asymptotic values as $x \rightarrow \pm \infty$ have been assumed. We have found that in such cases the variable transformation, $d z=l(x)^{1 / 2} d x$, is effective. First, for an adiabatic model where $l(x)$ changes very slowly compared with the soliton size, we have obtained the conditions of whether a soliton is transmitted or reflected from discussions of energy conservation. Next, for nonadiabatic cases, the perturbation theory of McLaughlin and Scott has been applied to our system. For two cases of an abruptly changed $l(x)$ and of a gradually changed $l(x)$, the time evolutions of the soliton parameters (the velocity and position parameters) have been solved. Radiations have also been evaluated by solving the first-order equation of the perturbation. Not only radiation wave forms but also radiation energies have been compared with those of the direct numerical integration of the original partial differential equation. Agreement between them is surprisingly good.

\section{ACKNOWLEDGMENTS}

One of us (S.S.) expresses his sincere thanks to the hospitality of the personnel in Physics Laboratory $I$, the Technical University of Denmark, during his stay there. We would like to acknowledge A. C. Scott for his thoughtful comments at the last stage of this work.
*Permanent address: Electrotechnical Laboratory, 1-1-4, Umezono, Sakura-mura, Niihari-gun, Ibaraki 305 Japan.

${ }^{1}$ A. C. Scott, Am. J. Phys. 37, 52 (1969).

${ }^{2}$ T. A. Fulton, R. C. Dynes, and P. W. Anderson, Proc. IEEE 61, 28 (1973).

${ }^{3}$ S. Sakai, H. Akoh, and H. Hayakawa, Jpn. J. Appl. Phys. 24,
L771 (1985).

${ }^{4}$ D. W. McLaughlin and A. C. Scott, Phys. Rev. A 18, 1652 (1978).

${ }^{5}$ International Mathematical and Statistical Library, 9th ed. (IMSL, Houston, 1982) (unpublished). 